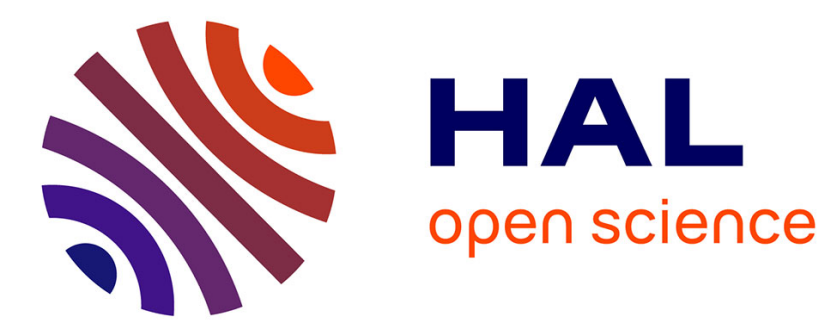

\title{
Extended full-field optical coherence microscopy
}

\author{
Arnaud Dubois
}

\section{To cite this version:}

Arnaud Dubois. Extended full-field optical coherence microscopy. 3rd International Topical Meeting on Optical Sensing and Artificial Vision, May 2012, Saint-Petersbourg, Russia. pp.123-132. hal00713696

\section{HAL Id: hal-00713696 \\ https://hal-iogs.archives-ouvertes.fr/hal-00713696}

Submitted on 2 Jul 2012

HAL is a multi-disciplinary open access archive for the deposit and dissemination of scientific research documents, whether they are published or not. The documents may come from teaching and research institutions in France or abroad, or from public or private research centers.
L'archive ouverte pluridisciplinaire HAL, est destinée au dépôt et à la diffusion de documents scientifiques de niveau recherche, publiés ou non, émanant des établissements d'enseignement et de recherche français ou étrangers, des laboratoires publics ou privés. 


\title{
Extended full-field optical coherence microscopy
}

\author{
Arnaud Dubois \\ Institut d'Optique Graduate School, Laboratoire Charles Fabry, \\ CNRS UMR 8501,Univ. Paris-Sud, 2 avenue Augustin Fresnel, 91127 Palaiseau Cedex, France
}

\begin{abstract}
Full-field optical coherence microscopy (FF-OCM) is a recent optical technology based on low-coherence interference microscopy for semi-transparent sample imaging with $\sim 1 \mu \mathrm{m}$ spatial resolution. FF-OCM has been successfully applied to three-dimensional imaging of various biological tissues at cellular-level resolution. The contrast of FF-OCM images results from the intensity of light backscattered by the sample microstructures. This contrast mechanism, based on refractive index changes, provides information on the internal architectural morphology of the sample. In this paper, we present a multimodal FF-OCM system, capable of measuring simultaneously the intensity, the power spectrum and the phase-retardation of light backscattered by the sample being imaged. Tomographic fluorescencebased images can also be produced by coupling to the FF-OCM set-up a fluorescence microscopy system with structured illumination. Fluorescence targeted probes can be used to identify molecular components of subcellular scattering structures. Compared to conventional FF-OCM, this multimodal system provides enhanced imaging contrasts at the price of a moderate increase in experimental complexity and cost.
\end{abstract}

Keywords: Optical coherence tomography, interference microscopy, fluorescence microscopy, polarimetry, spectroscopy PACS: 87.64.M-, 87.64.Kv, 87.64.K-, 87.85.Pq

\section{INTRODUCTION}

Optical coherence tomography (OCT) is a scanning optical imaging technique with micrometer-scale resolution, based on low-coherence interferometry [1-2]. OCT has been established in a variety of biomedical applications [3-5] and for material characterization [6]. OCT measures the intensity of light backscattered by the internal structures of the sample being imaged. Several extensions to the OCT technique have been developed to provide additional information. By measuring the temporal phase change of backscattered light, Doppler-OCT provides information about the speed of moving backscattering particles within the sample $[7,8]$. Polarization-sensitive OCT uses the information carried by the polarization state of backscattered light to improve the imaging contrast [9-11]. Spectroscopic OCT provides spatially-resolved measurements of the power spectrum of backscattered light by detection and processing of the whole interferometric signal [12-14]. Fluorescence imaging is another imaging modality that has been combined with OCT to provide information on the biochemical properties of the sample being imaged using endogenous or exogenous fluorophores $[15,16]$.

Full-field optical coherence microscopy (FF-OCM) is an alternative technique to scanning OCT, based on white-light interference microscopy [17-19]. FF-OCM produces en face tomographic images obtained by arithmetic combination of interferometric images acquired with an area camera. The whole field of view is illuminated with low-coherence light (temporally and spatially). The major interest for FF-OCM is its ultra-high imaging resolution in both transverse and axial directions using a simple and robust experimental arrangement [20-21]. Conventional FF-OCM has been applied in a variety of biomedical application [22-25]. Several extensions to the technique have also been proposed to provide additional information on the birefringence [26] or the spectroscopic properties [27] of the sample. Fluorescence microscopy was recently coupled to FFOCM to yield complementary morphological and functional information [28].

We present in this paper a multimodal FF-OCM system [29], capable of measuring simultaneously the power spectrum, the intensity and the birefringence-induced phase retardation of backscattered light. Moreover, a full-field tomographic fluorescence imaging modality can be coupled to the FF-OCM system. Images of several samples are presented to illustrate the performance of this extended multimodal imaging system.

\section{SPECTROSCOPIC POLARIZATION-SENSITIVE FF-OCM}

\section{Experimental setup}

The layout of the multimodal FF-OCM imaging system is shown in Figure 1. 


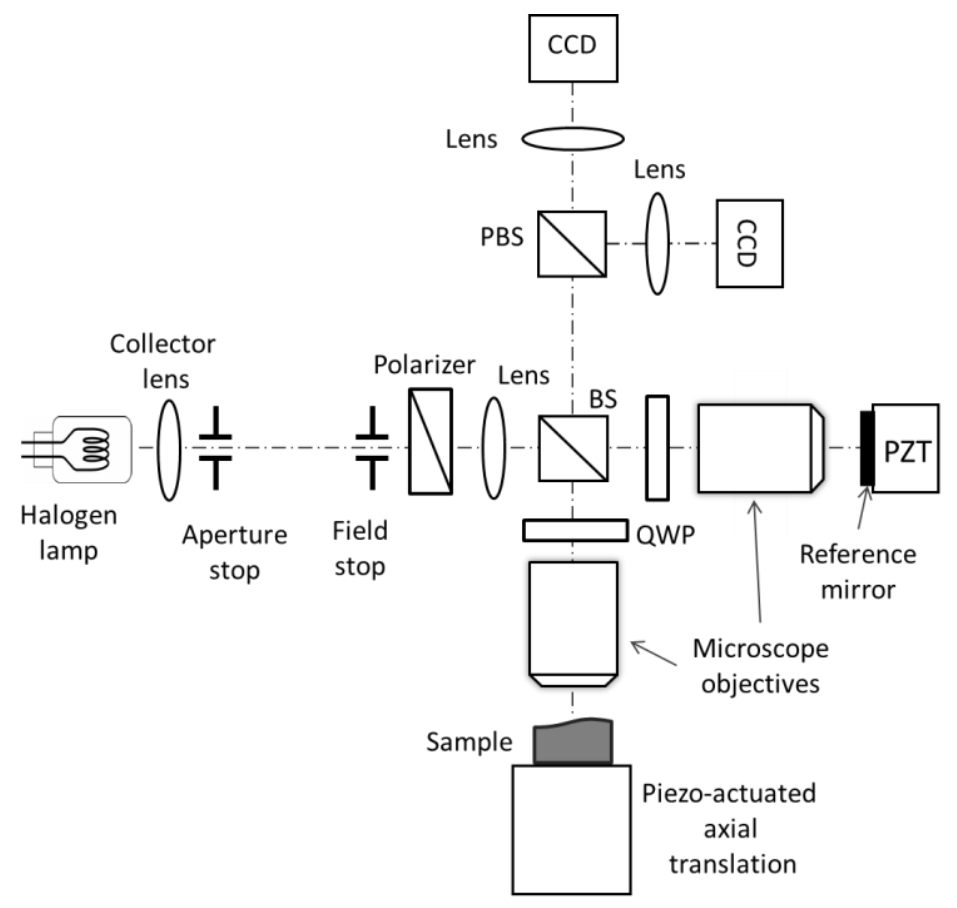

FIGURE 1. Experimental setup of multimodal FF-OCM for simultaneous intensity, phase-retardation and power spectrum measurements. The PZT is activated for real-time 2D en-face imaging (intensity and phase-retardation simultaneously). QWP: quarter-wave plates (achromatic), BS: beam-splitter (broadband), PBS: polarizing beam-splitter (broadband).

A 100W halogen lamp is used as the low-coherence light source. It is set up in a Kohler illumination arrangement to achieve uniform illumination of the sample. The filament of the lamp is imaged onto an aperture stop by a collector lens. A lens $(f=150 \mathrm{~mm})$ relays this image onto the back focal plane of water-immersion microscope objectives. A broadband beam splitter (BS) separates the light beam into a reference arm and a sample arm of a Linnik interferometer. A reference mirror (2\% reflectivity) and a sample are placed in the focal planes of the microscope objectives. Identical water-immersion microscope objectives are employed. Light is linearly polarized at $0^{\circ}$ (//) before entering the interferometer using a Glan-Thomson polarizer. An achromatic quarter-wave plate (QWP) is placed in the sample arm at $45^{\circ}$, so that the sample is illuminate with circularly polarized light. Returning from the sample and passing again through the quarter-wave plate, light is in a new polarization state due to the optical anisotropy properties of the sample. Another achromatic quarter-wave plate is placed in the reference arm at $22.5^{\circ}$, so that reference light at the interferometer output is linearly polarized at a $45^{\circ}$ angle to the incident polarization, and has equal intensity in the two orthogonal polarization states // and $\perp$. A broadband polarizing beam-splitter (PBS), placed at the output of the microscope, splits the interferometric image in two images with orthogonal polarizations oriented at $0^{\circ}(/ /)$ and $90^{\circ}$ $(\perp)$ with respect to the incident polarization. These images are projected onto two identical Charge-Coupled Devices (CCD) (DALSA 1M15, 1024×1024 pixels, $15 \mathrm{~Hz}$ ) using identical achromatic doublets of focal length equal to $400 \mathrm{~mm}$. $2 \times 2$ pixel binning is applied to increase the dynamic range of the detection. This $2 \times 2$ pixel binning is performed numerically after each image acquisition. The resulting images of 512×512 pixels are saved in 16 bit files on the computer hard disk for processing. A subnanometer-resolution piezoelectric-actuated axial translation stage is used to displace the sample along the optical axis with a step of a few nanometers in order to acquire the full interferometric signal. Two stacks of interferometric images are thus acquired simultaneously by the two CCDs. After acquisition, the stacks are processed to extract the power spectrum, the intensity and the birefringence-induced phase-retardation of light backscattered by the sample. Three-dimensional (3D) images with three distinct contrasts can thus be produced. Due to the relatively long acquisition times, the interferometer is placed in a box to prevent from turbulence and the whole system is installed on an active vibration isolation table.

If spectroscopic measurements are not desired, high-speed en face imaging with intensity and phase-retardation contrasts is possible, using fast phase-shifting interferometry by making the reference surface oscillate, as described in [26,29]. The oscillation of the reference mirror is achieved by using a piezo actuator (PZT). 


\section{Principle of operation}

\section{Acquisition of the interferometric data}

The sample is displaced, using the high-resolution piezo-actuated translation stage, in the axial direction in steps small enough to resolve the interference fringes. The displacement increment is adjusted to $\delta=35 \mathrm{~nm}$, which corresponds to a phase change in the interferometer of $4 \pi \times n_{i n} \times \delta / \lambda \sim \pi / 4$, where $n_{i m}$ is the refractive index of the objective immersion medium and $\lambda=750 \mathrm{~nm}$ is the effective center wavelength. At each step $k, N_{\text {acc }}$ images are acquired by each CCD and are summed up in order to increase the dynamic range and hence the detection sensitivity. Two $z$-stacks of interferometric images, $E_{k}^{\perp}$ and $E_{k}^{\prime \prime}$, with orthogonal polarizations are thus acquired and saved on the computer hard disk.

\section{Calculation of intensity and phase retardation}

Intensity and phase-retardation images are extracted from the stacks of interferometric images acquired by the cameras. The method consists of combining several interferometric images with a relative phase-shift. An algorithm with a phase-shift of $\pi / 2$ between successive images is used [29]. A stack of en face intensity-based tomographic images of the sample at sucessive depths $z=k \delta$, denoted as $R_{\text {sample }}$, is calculated from the following image combination:

$$
R_{\text {sample }}=\left[\left(E_{k}^{\perp}-E_{k+4}^{\perp}\right)^{2}+\left(E_{k+2}^{\perp}-E_{k+6}^{\perp}\right)^{2}+\left(E_{k}^{\prime \prime}-E_{k+4}^{\prime \prime}\right)^{2}+\left(E_{k+2}^{\prime \prime}-E_{k+6}^{\prime \prime}\right)^{2}\right] .
$$

The phase-retardation of light backscattered by the sample structures located at depth $z=k \delta$ is calculated from:

$$
\phi=\operatorname{atan} \sqrt{\frac{\left(E_{k}^{\prime \prime}-E_{k+4}^{\prime \prime}\right)^{2}+\left(E_{k+2}^{\prime \prime}-E_{k+6}^{\prime \prime}\right)^{2}}{\left(E_{k}^{\perp}-E_{k+4}^{\perp}\right)^{2}+\left(E_{k+2}^{\perp}-E_{k+6}^{\perp}\right)^{2}}} .
$$

It has to be noted that the measured phase-retardation $\phi$ results from the accumulation of phase changes occurring during the propagation of light in the sample from the surface to the imaging depth and back to the surface. By applying Eq. (2) for all the values of the index $k$, a stack of en face phase-retardation images is obtained.

\section{Calculation of the spectroscopic information}

Power spectra are obtained by Fourier analysis of the stack of interferometric images $E^{\perp}$ acquired by one of the CCD cameras, as described in [27]. A Gaussian-shaped window is multiplied with the interferometric data to isolate local interferograms. Spatially-resolved power spectra are then obtained from the FFT calculation of these local interferograms. The window is moved axially to obtain power spectra at different depths. It has to be noted, however, that the spectral information measured at an arbitrary depth in the sample where scattering occurs is not strictly spatially-resolved since it may be modified by the effects of absorption and scattering of light during its propagation from the surface to this depth and back to the surface. The choice of the width of the Gaussian window results in a trade-off between spectral resolution and spatial resolution of the spectral data. A Gaussian window with width equal to the imaging axial resolution, i.e. a full width at half maximum (FWHM) of $1.0 \mu \mathrm{m}$, was chosen. The spectral resolution, defined as the FWHM of the Fourier transform of the Gaussian window, is equal to $240 \mathrm{~nm}$. Despite a low spectral resolution, spectral shifts can be measured with a sensitivity of a few nanometers on an immobile sample depending on the signal-to-noise ratio [27]. After the calculation of local spectra associated to each point of the imaged volume, it is necessary to quantify some aspect of the spectra for display. Due to the low spectral resolution, the measured spectra are always peak functions. An appropriate spectroscopic metric is then the spectrum center of mass.

Alternative methods for accessing spectroscopic information in FF-OCM without post-processing, based on wavelength demultiplexing and parallel detection, have been demonstrated or suggested [30,31]. These methods are insensitive to scan nonlinearities or sample movements and can therefore potentially provide better sensitivity and accuracy. They are however technically more difficult to implement. On the contrary, the application of Fourier-transform spectroscopy in FF-OCM is straightforward since this technique involves a Michelson-type interferometer. 


\section{Performance}

\section{Intensity detection sensitivity}

The intensity detection sensitivity of the multimodal FF-OCM system can be established assuming that the shot-noise is the predominant source of noise [29]. The smallest detectable reflectivity $R_{\min }$ is then given by

$$
R_{\min }=\frac{\left(R_{r e f}+R_{i n c}\right)^{2}}{4 N_{a c c} \xi_{s a t} R_{r e f}} .
$$

As can be seen from Eq. (3), the detection sensitivity depends on several experimental parameters. The full-well capacity $\xi_{\text {sat }}$ of the camera pixels has to be as large as possible. By accumulating a number $N_{a c c}$ of images, the detection sensitivity can be improved. The proportion of light that does not interfere, represented by the equivalent reflectivity coefficient $R_{\text {inc }}$, must be as low as possible. In practice the minimal value of $R_{i n c}$ is imposed by the sample itself. By calculating the derivative of Eq. (3) with respect to $R_{r e f}$, one can see that the optimal reflectivity of the reference mirror is $R_{r e f}=R_{i n c}$. This optimal value therefore depends on the sample being imaged; it does not exceed a few percents for most biological tissues. According to Eq. (3), a detection sensitivity $-10 \times \log \left(R_{\text {min }}\right)$ on the order $90 \mathrm{~dB}$ can be obtained in an acquisition time of $\sim 3 \mathrm{~s}\left(R_{\text {ref }}=0.02, R_{\text {inc }}=0.01\right.$, $N_{a c c}=10, \xi_{\text {sat }}=10^{6}$ ).

A particularly interesting feature of FF-OCM is that the detection sensitivity does not depend on the illumination bandwidth. This is an advantage over time-domain scanning OCT, where detection sensitivity and illumination bandwidth are inversely proportional [32]. In frequency-domain OCT, the illumination bandwidth does not affect the detection sensitivity as well [33]. This latter technique achieves the best trade-off between detection sensitivity and acquisition speed along with high axial resolution [34]. On the other hand, the transverse resolution of frequency-domain OCT is limited to 10-20 $\mu \mathrm{m}$, which is one order of magnitude larger than the transverse resolution that can be achieved with FF-OCM as will be shown.

\section{Spatial resolution}

The spatial resolution of an imaging system can be characterized by the image of a point, i.e. the so-called point-spread function (PSF). In FF-OCM, the PSF is determined by the properties of both the microscope objectives and the power spectrum of the detected light.

The theoretical transverse resolution of FF-OCM depends on the center optical wavelength $\lambda$ and the numerical aperture $N A$ of the microscope objectives. The effective center wavelength being $\lambda=750 \mathrm{~nm}$, the theoretical transverse resolution, at the surface of the sample, is $\sim 1 \mu \mathrm{m}$ when microscope objectives with numerical aperture of $N A=0.3$ are employed. We mention that a degradation of the resolution occurs progressively when the imaging depth increases due to refractive index mismatch between the sample and the immersion medium. This effect is common to all microscopy systems and may become dramatic when a high numerical aperture is employed. In comparison, the transverse resolution of conventional OCT is 10-20 times lower since this technique produces cross-sectional images (B-scans), which requires a depth of focus equal to the axial extent of the images and therefore a low numerical aperture.

The axial resolution of FF-OCM is determined theoretically by both the effective coherence length and the depth of focus of the microscope objectives [17]. The FWHM of the axial response, at the surface of the sample, is given by

$$
\Delta z=\left[\frac{N A^{2}}{n_{i m} \lambda}+\frac{n_{i m} \pi}{2 \ln 2}\left(\frac{\Delta \lambda}{\lambda^{2}}\right)\right]^{-1}
$$

where $n_{i m}$ is the refractive index of the objective immersion medium and $\Delta \lambda$ the effective source spectral bandwidth. When objectives with relatively low numerical aperture are employed, the axial resolution is imposed by the ultra-short coherence length of the halogen lamp. The axial resolution is $\sim 1 \mu \mathrm{m}$. This ultrahigh axial resolution degrades with the imaging depth due to dispersion mismatch between the interferometer arms, although this mismatch is minimized by using water-immersion microscope objectives. In comparison with ultra-short femtosecond lasers or supercontinuum fiber lasers used for ultrahigh- 
resolution OCT [35, 36], a thermal light source has a smoother spectrum that does not contain spikes or emission lines that could cause side lobes in the coherence function and create artifacts in the images.

\section{Acquisition times}

Two stacks of interferometric images need to be acquired to obtain the spectroscopic information. In practice, the entire accessible imaging depth of the sample is scanned. For example, the acquisition of two stacks of accumulated interferometric images $\left(N_{a c c}=10\right)$ from the surface of the sample down to a depth of $200 \mu \mathrm{m}$ represents an acquisition time of $\sim 70 \mathrm{~min}$. This long acquisition time is limited by the CCD frame rate and full-well-capacity, and also by the time spent accessing and writing the data on the computer hard disk. Because of the acquisition time, the technique is currently not suitable for in vivo imaging, and care must also be taken to avoid mechanical instabilities in the interferometer. Other approaches for accessing spectroscopic information, based on wavelength de-multiplexing and parallel detection [30, 31], are much faster than the Fourier-analysis method used here. These approaches therefore require less care for mechanical and thermal stability and are more appropriate for in vivo applications. Their implementation in FF-OCM is however not so straightforward. We can reasonably expect to reduce the acquisition time of our FF-OCM system by a factor of at least ten by using the latest generations of high dynamic CMOS cameras and fast computers.

If spectroscopic measurements are not desired, it is possible to obtain intensity and phase-retardation in a much shorter time, since it is not necessary to acquire the full interferometric signal, i.e. to resolve the interference fringes. Only the amplitude of the interference signal in two orthogonal polarization states needs to be measured. Combinations of 4 pairs of interferometric images (i.e. a total of 8 images; each camera acquires 4 images), yield an en face image with intensity and phase-retardation information, the phase being shifted in the interferometer between each image pair acquisition, as explained in [26]. The phase-shift is achieved by making the reference mirror oscillate, using a piezoelectric actuator (see Fig. 1), at a frequency equals to one quarter of the CCD frame rate (i.e. $3.75 \mathrm{~Hz}$ ). The CCD cameras are synchronized with the PZT oscillation to acquire 4 images per modulation period. With our cameras, the acquisition time to produce an en face image with both intensity contrast and phaseretardation contrast is about 3 seconds (with $N_{a c c}=10$ ). This time could even be reduced by using other cameras.

\section{Examples of images}

To illustrate the performance of the multimodal FF-OCM system, a Xenopus Laevis tadpole was imaged (ex-vivo). The tadpole was placed in a tank filled with phosphate-buffered saline (PBS, $\mathrm{pH} 7$ ) for animal preservation and to provide index matching. A stack of 5000 images was recorded, which represents a 3D data-set of 512×512×5000 elements ( 5 GigaBytes). The physical size of the imaged volume is $530 \mu \mathrm{m} \times 530 \mu \mathrm{m} \times 175 \mu \mathrm{m}(x \times y \times z)$. A cross-sectional image extracted from the 3D data is shown in Figure 2. For simultaneously displaying intensity and spectroscopic information, we use a HSL (hue, saturation, luminance) color map. Hue encodes the spectroscopic information (spectra center of mass position), whereas luminance and saturation encode the intensity information. We restrict a Hue variation from 0 to 0.5 (the full available hue range being from 0 to 1 ), so that the color vary from red to light blue. Luminance ranges from 0 to 1 and saturation from 0.5 to 1 . The intensity information reveals the internal tissue morphology. Pleomorphic mesenchymal cells can be visualized. Cell membranes, cell nuclei, and melanocytes appear highly backscattering compared to cytoplasm. The spectroscopic information reveals a combination of absorption and scattering. In particular, melanocytes appear red, indicating that they absorb more short wavelength than long wavelength.

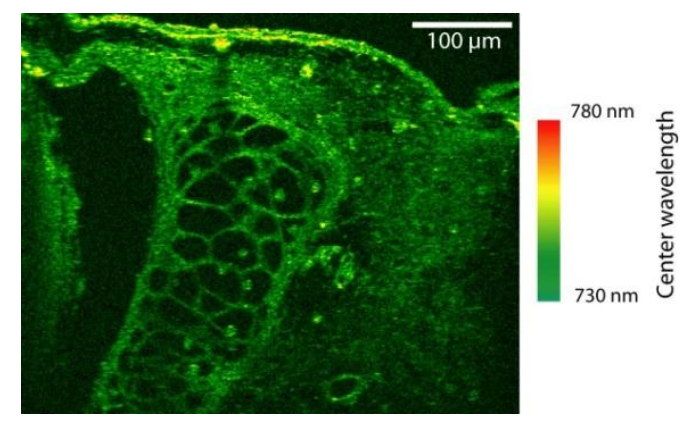

FIGURE 2. Cross-sectional combined intensity/spectroscopic FF-OCM images of an ex-vivo Xenopus Laevis tadpole embryo. 
Bovine tendon was imaged to illustrate the performance of the system to reveal the presence of birefringence in biological tissues. The sample was imaged $48 \mathrm{~h}$ after the bovine death. The sample was placed in a tank filled with phosphate-buffered saline (PBS, pH 7) for tissue preservation and to provide index matching. Figure 3 shows reconstructed cross-sectional images. Image A, represented in logarithmic scale, corresponds to the conventional intensity-based image computed with Eq. (1). The associated phase retardation image, calculated with Eq. (2), is shown in B. The banded structure reveals the birefringence of the tissue resulting from the presence of collagen.

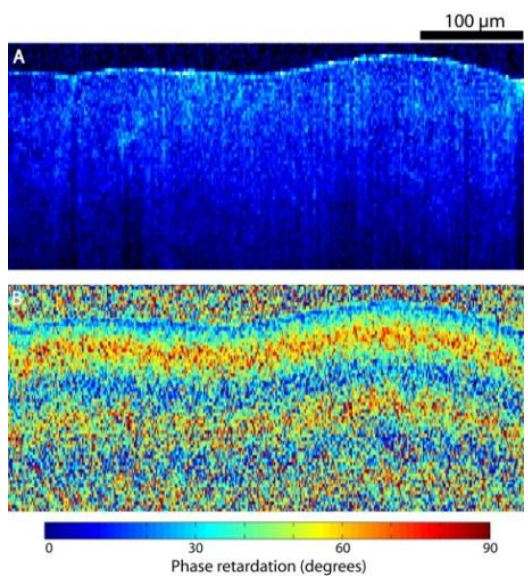

FIGURE 3. Cross-sectional images of fresh bovin tendon. (A) Conventional intensity-based image in logarithmic scale. (B) Phase retardation image.

\section{COMBINED FF-OCM / FLUORESCENCE MICROSCOPY}

\section{Experimental setup}

The experimental setup of combined FF-OCM / fluorescence microscopy is represented schematically in Figure 4. A CW solid-state laser (Coherent, 488-200 CRDM) emitting at 488-nm is used for the fluorescence imaging modality. The laser beam is coupled into the system by a dichroic beam splitter that reflects visible light $(\lambda<700 \mathrm{~nm})$ and transmits infrared light $(\lambda>700 \mathrm{~nm})$. A rotary diffuser is placed in the path of the laser beam to reduce the effect of speckle. A lens $(f=100 \mathrm{~mm})$ is used to expand the size of the illumination field on the sample. Visible fluorescence light $(\lambda<650 \mathrm{~nm})$ emitted by the sample and collected by the microscope objective is reflected by the dichroic beam splitter and focused onto a CCD detector (Photometrics, CoolSnap HQ, 1392×1040 pixels) by an achromatic doublet lens $(f=300 \mathrm{~mm}$ ). A notch filter (Semrock, NF-488) is used to suppress the excitation light. Water-immersion microscope objectives $(20 \mathrm{X}, N A=0.5)$ are employed. A 20 line pairs $/ \mathrm{mm}$ grid is placed in the laser beam path and is imaged on the field stop plane that is conjugate of the sample plane. The so-called structured illumination microscopy technique is thus implemented here [37]. The grid is mounted on a piezo-actuated translation stage (PZT) that controls its transverse positioning. Images of the sample are acquired for three positions of the grid. These positions are shifted by one third of the grid period with respect to each other. The acquired images $I_{1}, I_{2}$ and $I_{3}$ are processed to get rid of the grid pattern. A conventional wide field fluorescence image is obtained from the algebraic summation of the three raw acquired images $I_{1}, I_{2}$ and $I_{3}$, whereas an optically sectioned image is obtained using the following image combination:

$$
I_{S}=\sqrt{\left(I_{1}-I_{2}\right)^{2}+\left(I_{1}-I_{3}\right)^{2}+\left(I_{2}-I_{3}\right)^{2}} .
$$

The images obtained from the FF-OCM imaging modality and the optically-sectioned fluorescence imaging modality can be displayed separately or overlaid. The detectors used in the two imaging modalities have different format in terms of pixel number and pixel size. To overlay the fluorescence and FF-OCM images, it was necessary to crop and then interpolate the initial FFOCM image so that it shows the same field of view and has the same number of data points as the fluorescence image. 


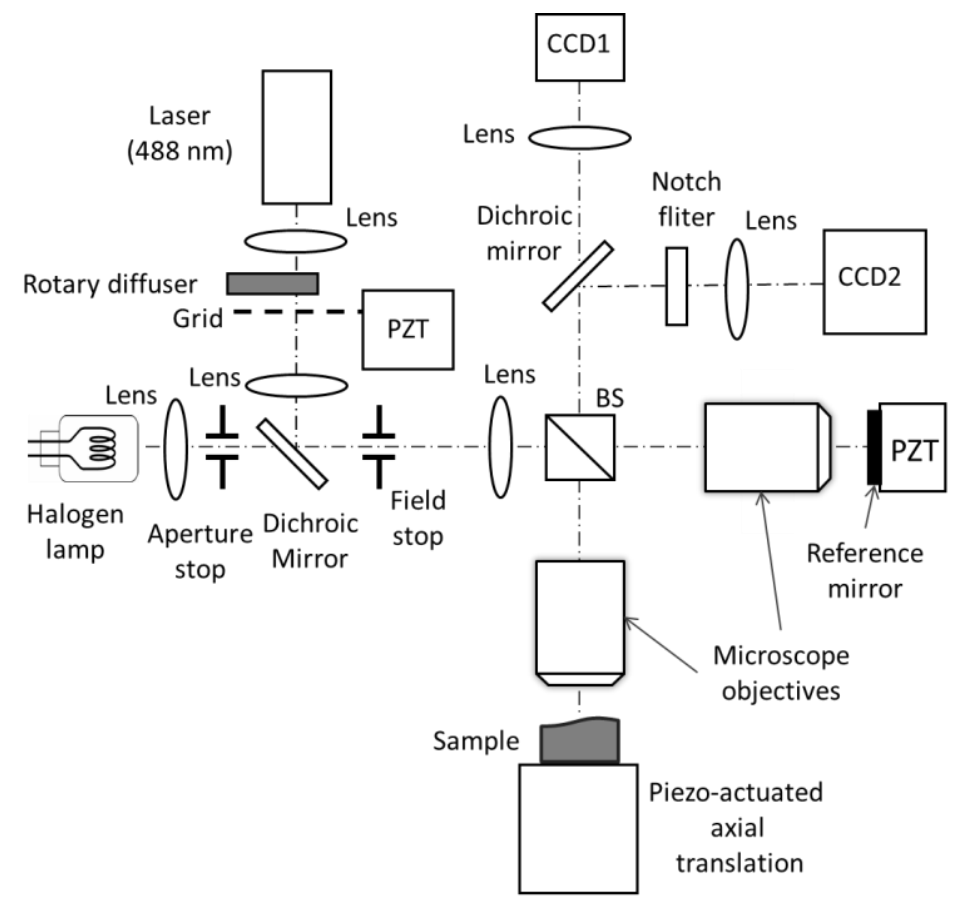

FIGURE 4. Experimental setup of combined FF-OCM / fluorescence microscopy. BS: beamsplitter (broadband), PZT: piezoelectric transducer, $\mathrm{CCD}$ : charge-coupled device.

\section{Performance}

\section{Spatial resolution and detection sensitivity}

The coherence function associated with the halogen lamp source was determined experimentally. Its FWHM gives the axial resolution of the FF-OCM imaging modality and was measured to be $1.5 \mu \mathrm{m}$ in air $(1.1 \mu \mathrm{m}$ in tissue assuming a refractive index of 1.4). The theoretical lateral resolution is $0.9 \mu \mathrm{m}$ at $800 \mathrm{~nm}$ center wavelength. A typical number of $10 \mathrm{FF}-\mathrm{OCM}$ images are acquired and averaged to increase the dynamic range. The detection sensitivity was measured to be $89 \mathrm{~dB}$. The fluorescence PSF was measured by imaging fluorescent beads. With $0.5 \mu \mathrm{m}$ diameter beads, a lateral resolution of $0.7 \mu \mathrm{m}$ was determined, which is comparable to the theoretical value of $0.6 \mu \mathrm{m}$ at a wavelength of $520 \mathrm{~nm}$. A z-stack of images of $1 \mu \mathrm{m}$ diameter beads was acquired to evaluate the axial resolution. A value of $6.0 \mu \mathrm{m}$ was measured. A $4.5 \mu \mathrm{m}$ theoretical axial resolution was computed based on the axial response function derived in [37]. The discrepancy between measured and theoretical values is attributed to the presence of optical aberration in the imaging system, including the aberration associated with the imaging of the grid into the sample. Simultaneous acquisition of a fluorescence image and a FF-OCM image takes $2.7 \mathrm{~s}$.

\section{Illustration of the imaging capability}

Mouse colon tissues have been imaged one hour after the mouse death. Prior to imaging, the tissues were stained with acridine orange $(\mathrm{AO})$ and set between a glass slide and a coverslip. $\mathrm{AO}$ is a nucleic acid stain with dual peak emission wavelengths around $520 \mathrm{~nm}$ and $650 \mathrm{~nm}$ when bound to DNA and RNA, respectively. A drop of distilled water was deposited on the coverslip and served as the immersion medium for the microscope objective. Figures 5(a) and 5(b) show the conventional and the optically sectioned fluorescence images, respectively. Most out-of-focus light is rejected in the optically sectioned image. The FF-OCM image is shown in Fig. 5(c). The optically sectioned fluorescence image and the FF-OCM image are overlaid in Fig. 5(d) for direct comparison. The imaging depth was about $20 \mu \mathrm{m}$ below the tissue surface. The fluorescence and FF-OCM images are totally different. The FF-OCM image provides morphological information on the tissue, whereas the fluorescence image reveals the position of individual cell nuclei. 

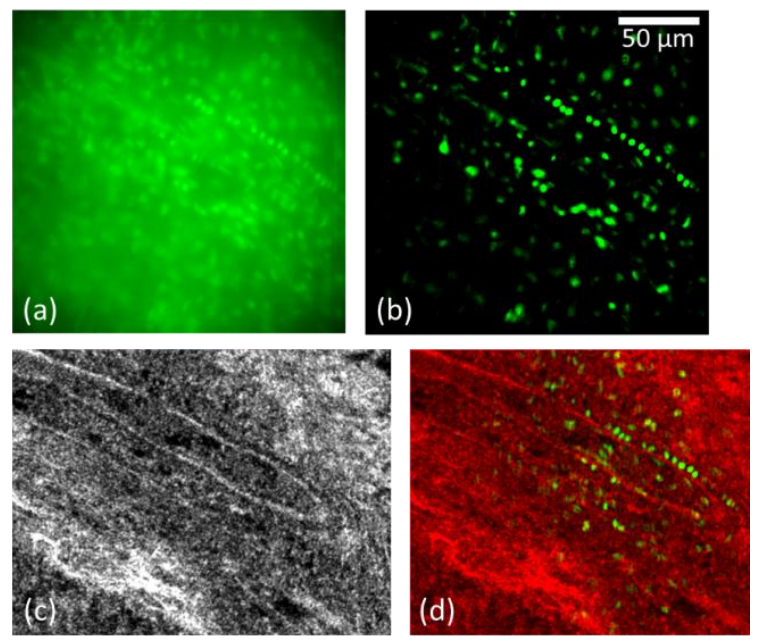

FIGURE 5. (a) Conventional fluorescence image, (b) optically sectioned fluorescence image and (c) FF-OCM image of mouse colon stained with AO; (d) superposition of (b) and (c) in pseudo-colors.

Other target-specific fluorophores could be used. Microscope objectives with higher $N A$ could also be employed to improve the lateral resolution in both modalities and the axial resolution of the fluorescence imaging technique. Simultaneous acquisition enables capturing an image of the same tissue location regardless of sample motion. However this motion needs to be negligible during the acquisition time to avoid image blurring. Overlaying the images obtained from each imaging modality highlights their differences.

The imaging depth is limited by tissue scattering and absorption. The fluorescence modality dictates the maximum imaging depth while being able to co-register a fluorescence image and an FF-OCM image at the same plane location in a specimen. This is because visible light is more scattered than infrared light in biological tissues [38] and intensity-based detection techniques are less sensitive than interferometric detection techniques [39].

\section{CONCLUSIONS}

A multimodal FF-OCM system was demonstrated, capable of measuring simultaneously the intensity, the power spectrum and the phase-retardation of light backscattered by the sample being imaged, with $\sim 1 \mu \mathrm{m}$ spatial resolution. The intensity-based images provide information on the internal architectural morphology of the sample. Spectroscopic imaging, possible over a wavelength range from 600 to $900 \mathrm{~nm}$, provides local information on the tissue absorption and scattering properties. The polarization-sensitive imaging modality measures the phase retardation of light backscattered by the sample micro-structures. Birefringent tissues can thus be easily distinguished from other tissues.

Fluorescence microscopy can be coupled to FF-OCM for providing high resolution FF-OCM images and fluorescence images simultaneously. Optical sectioning is achieved in the fluorescence modality by using a structured illumination technique. The architecture of the system allows one to obtain full-field images without raster scanning in both modities. The combination of the data types obtained from each imaging modality provides complementary information and could be useful for pathological diagnosis and studying cellular function based on tissue morphology and biochemical properties.

An integrated system capable of multiple contrast imaging can potentially be valuable in biomedical applications by providing complementary information during biological tissue screening. This has the potential for better understanding cellular functions, and improving the ability to detect pathologies by monitoring morphological and biochemical changes in tissues. This extended multimodal imaging system could be used in conjunction with biopsy procedures. Excised tissue samples could be explored with minimal preparation as opposed to standard histopathology process. 


\section{ACKNOWLEDGMENTS}

The authors wish to acknowledge the funding agency Réseau Thématique de Recherche Avancée. They are grateful to C. Martin and F. Pain for providing the animal tissues. Institut d'Optique Graduate School is a member of the European network Photonics4Life.

\section{REFERENCES}

1. D. Huang, E. A. Swanson, C. P. Lin, J. S. Schuman, W. G. Stinson, W. Chang, M. R. Hee, T. Flotte, K. Gregory, C. A. Puliafito, and J. G. Fujimoto, "Optical coherence tomography," Science 254, 1178-1181 (1991).

2. A. F. Fercher, "Optical coherence tomography," J. Biomed. Opt. 1, 157-173 (1996).

3. E. A. Swanson, J. A. Izatt, M. R. Hee, D. Huang, C. P. Lin, J. S. Schuman, C. A. Puliafito, and J. G. Fujimoto, "In-vivo retinal imaging by optical coherence tomography," Opt. Lett. 18, 1864-1866 (1993).

4. M. Wojtkowski, R. Leitgeb, A. Kowalczyk, T. Bajraszewski, and A. F. Fercher, "In-vivo human retinal imaging by Fourier domain optical coherence tomography," J. Biomed. Opt. 7, 457-463 (2002).

5. J. G. Fujimoto, "Optical coherence tomography for ultrahigh resolution in vivo imaging," Nat. Biotechnol. 21, 1361-1367 (2003).

6. K. Wiesauer, M. Pircher, E. Götzinger, S. Bauer, R. Engelke, G. Ahrens, G. Grützner, C.Hitzenberger, and D.Stifter, "En-face scanning optical coherence tomography with ultra-high resolution for material investigation," Opt. Express 13, 1015-1024 (2005).

7. X. J. Wang, T. E. Milner, and J. S. Nelson, "Characterization of fluid flow velocity by optical Doppler tomography," Opt. Lett. 20, 1337-1339 (1995)

8. J. A. Izatt, M. D. Kulkarni, S. Yazdanfar, J. K. Barton, and A. J. Welch, "In vivo bidirectional color Doppler flow imaging of picoliter blood volumes using optical coherence tomography,” Opt. Lett. 22, 1439-1441 (1997).

9. J. F. De Boer, T. E. Milner, M. J. C Van Gemert, and J. S. Nelson,"Two-dimensional birefringence imaging in biological tissue by polarization-sensitive optical coherence tomography," Opt. Lett. 22, 934-936 (1997).

10. C. K. Hitzenberger, E. Götzinger, M. Sticker, and A. F. Fercher, "Measurement and imaging of birefringence and optic axis orientation by phase resolved polarization sensitive optical coherence tomography," Opt. Express 9, 780-790 (2001).

11. K. Wiesauer, M. Pircher, E. Goetzinger, C. K. Hitzenberger, R. Engelke, G Ahrens, G. Gruetzner, and D. Stifter, "Transversal ultrahigh-resolution polarization sensitive optical coherence tomography for strain mapping in materials," Opt. Express 14, 5945-5953 (2006).

12. U. Morgner,W. Drexler, F. X. Kärtner, X. D. Li, C. Pitris, E. P. Ippen, and J. G. Fujimoto. "Spectroscopic optical coherence tomography," Opt. Lett. 25, 111-113 (2000).

13. R. Leitgeb, M. Wojtkowski, A. Kowalczyk, C. K. Hitzenberger, M. Sticker, and A. F. Fercher, "Spectral measurement of absorption by spectroscopic frequency-domain optical coherence tomography," Opt. Lett. 25, 820-822 (2000).

14. D. Adler, T. Ko, P. Herz, and J. G. Fujimoto, "Optical coherence tomography contrast enhancement using spectroscopic analysis with spectral autocorrelation,” Opt. Express 12, 5487-5501 (2004).

15. R. B. Rosen, M. Hathaway, J. Rogers, J. Pedro, P. Garcia, P. Laissue, G. M. Dobre and A. Gh. Podoleanu, "Multidimensional en-face OCT imaging of the retina," Opt. Express 17, 4112-4133 (2009).

16. S. Yuan, Q. Li, J. Jiang, A. Cable and Y. Chen, "Three-dimensional coregistered optical coherence tomography and line-scanning fluorescence laminar optical tomography,” Optics Letters 34, 1615-1617 (2009).

17. A. Dubois, L. Vabre, A. C. Boccara, and E. Beaurepaire, "High-resolution full-field optical coherence tomography with a Linnik microscope," Appl. Optics 41, 805-812 (2002).

18. L. Vabre, A. Dubois, and A.C. Boccara, “Thermal-light full-field optical coherence tomography,” Opt. Lett. 27, $530-533$ (2002).

19. B. Laude, A. De Martino, B. Drévillon, L. Benattar, and L. Schwartz, "Full-field optical coherence tomography with thermal light," Appl. Opt. 41, 6637-6645 (2002).

20. A. Dubois, K. Grieve, G. Moneron, R. Lecaque, L. Vabre, A.C. Boccara, "Ultrahigh-resolution full-field optical coherence tomography," Appl. Opt. 43, 2874-2882 (2004).

21. A. Dubois, G. Moneron, K. Grieve, A.C. Boccara, "Three-dimensional cellular-level imaging using full-field optical coherence tomography,” Phys. Med. Biol. 49, 1227-1234 (2004).

22. A. Perea-Gomez, A. Moreau, A. Camus, K. Grieve, G. Moneron, A. Dubois, C. Cibert, J. Collignon, "Initiation of gastrulation in the mouse embryo is preceded by an apparent shift in the orientation of the anterior-posterior axis," Current Biol. 14, 197-207 (2004)

23. K. Grieve, M. Paques, A. Dubois, J. Sahel, A.C. Boccara, J.F. Le Gargasson, "Ocular tissue imaging using ultrahigh-resolution fullfield optical coherence tomography,” Invest. Ophthalmol. Visual Sci. 45, 4126-4131 (2004).

24. Y. Yang, A. Dubois, X. P. Qin, J. Li, A. El Haja, R. Wang, "Investigation of optical coherence tomography as an imaging modality in tissue engineering," Phys. Med. Biol. 51, 1649-1659 (2006).

25. M. Boccara, W. Schwartz, E. Guiot, G. Vidal, R. De Paepe, A. Dubois, A.C. Boccara, "Early chloroplastic alterations analysed by optical coherence tomography during harpin-induced hypersensitive response," The Plant Journal 50, 338-346 (2007)

26. G. Moneron, A.C. Boccara, A. Dubois, "Polarization-sensitive full-field optical coherence tomography," Opt. Lett. 32, 2058-2060 (2007).

27. A. Dubois, J. Moreau, A.C. Boccara, "Spectroscopic ultrahigh-resolution full-field optical coherence microscopy," Opt. Express 16, 17082-17091 (2008). 
28. H. Makhlouf, K. Perronet, G. Dupuis, S. Levêque-Fort, A. Dubois, "Simultaneous optically sectioned fluorescence and optical coherence microscopy with full-field illumination," Opt. Lett. 37, 1613-1615 (2012)

29. A. Dubois, "Spectroscopic polarization-sensitive full-field optical coherence microscopy," Opt. Express 20, $9962-9977$ (2012).

30. M. Laubscher, S. Bourquin, L. Froehly, B. Karamata, and T. Lasser, "Spectroscopic optical coherence tomography based on wavelength de-multiplexing and smart pixel array detection," Optics Comm. 237, 275-283 (2004).

31. I Abdulhalim, R. Friedman, L. Liraz, and R. Dadon, "Full-field frequency domain common path optical coherence tomography with annular aperture," Proc. of SPIE-OSA Biomedical Optics, SPIE 6627, 662719 (2007).

32. E. A. Swanson, D. Huang, M. R. Hee, J. G. Fujimoto, C. P. Lin, and C. A. Puliafito, "High-speed optical coherence domain reflectometry," Opt. Lett. 17, 151-153 (1992).

33. M. A. Choma, M. V. Sarunic, C. H. Yang, and J. A. Izatt, "Sensitivity advantage of swept source and Fourier domain optical coherence tomography," Opt. Express 11, 2183-2189 (2003).

34. R. Leitgeb, C. K. Hitzenberger, and A. F. Fercher, "Performance of fourier domain vs. time domain optical coherence tomography," Opt. Express 11, 889-894 (2003).

35. W. Drexler, U. Morgner, F. X. Kärtner, C. Pitris, S. A. Boppart, X. D. Li, E. P. Ippen, and J. G. Fujimoto, "In-vivo ultrahigh-resolution optical coherence tomography," Opt. Lett. 24, 1221-1223 (1999).

36. Y. Wang, Y. Zhao, J. S. Nelson, Z. Chen, and R. S. Windeler, "Ultrahigh-resolution optical coherence tomography by broadband continuum generation from a photonic crystal fiber," Opt. Lett. 28, 182-184 (2003).

37. M. A. Neil, R. Juskaitis, and T. Wilson, "Method of obtaining optical sectioning by using structured light in a conventional microscope," Opt. Lett. 22, 1905-1907 (1997).

38. P. Taroni, A. Pifferi, A. Torricelli, D. Comelli and R. Cubeddu, "In vivo absorption and scattering spectroscopy of biological tissues," Photochemicaland Photobiological Sciences 2, 124-129 (2003)

39. E. Beaurepaire, L. Moreaux, F. Amblard, and J. Mertz, "Combined scanning optical coherence and two-photon-excited fluorescence microscopy," Opt. Lett. 24, 969-971 (1999). 